\section{Arquitectura y virtualidad}

\section{Hacia una nueva condición material'}

Antoine Picon Proffesor de Havard University Craduate School of Design

\section{La naturalezam meramente formal de buena parte de la llamadad " "roduccí́n arqui-
tectonica digital" la ha alejado de otras discusiones, muchas de ellas centrales para nuestra cultura. Porque más alla de los blobs, la tecnologia CAD/CAM plantea una
evisión social y yolitica que compromete, ahora mas que nunca, a la arguitectura}

A menudo, el desarrollo de los medios digitales en cuanto herramie tas de diseño es presentado como amenaza a una de las dimensiones técnicas (es decir, su materialización). Un ejemplo es la preocupació
manifestada por Kenneth Frampton en sus últimos escritos, comenzando por Studies in tectonic culture. A pesar de los descargos expuestos por William Mitchell (Beckmann, 1998) y otros autores, se trata de un formalista de la producción de muchos arquitectos digitalas. El diseño
basado en las herramientas computacionales a menudo parece negar la dimensión material de la arquitectura y su profunda relación con trío pes-empuje-resistencia. En el monitor de un computador, las forma
parecen flotar libremente sin más restricciones parecen flotar libremente, sin más restricciones que las que imponen
imaginación del diseñador $y$ las posibilidades del software Hay algo profundamente inquietante en esa aparente libertad, que parece cuestionar nuestros supuestos más fundamentales respecto a la naturaleza de disciplina arquitectónica.

Sin embargo, idebemós suponer que el estado actual del diseño asistido

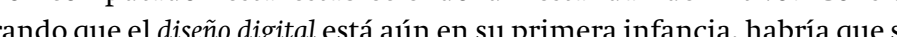
precavido en no aventurarse con conclusiones apresuradas basadas en aspectos que aún son pasajeros en él. Frampton y otros de sus detractores
tal vez asumen su condición actual como la definitiva, tomando dem siado en serio aspectos que aún están en evolución y subestimando de La presente tendencia a cierta inmaterialidad, o más bien una actitud a menudo simplista respecto a los materiales, puede por cierto ser pasa jera. Es más: lejos de ser puesta en peligro por el uso generalizado de computador y el desarrollo de ambientes virtuales, la materialidad prola producción arquitectónica. Se podría además especular si realmente el uso del computador, o el de sus extensiones como parte de una web. representan un alejamiento sustancial de los medios tradicionales de
la representación arquitectónica; en muchos sentidos, los dibujos bidmensionales producidos a mano no son más matéricos que los generado arquitectónica no necesariamente implica carencias materiales en su posterior realización.

Precisamente, me gustaría tomar esta pregunta genérica por la represenper los camb desarrollaré en este sentido es aquella que sostiene que la materialidad, como casi todos los aspectos de nuestro entorno, es fundamentalmente
una construcción cultural. Como sostienen varios exponentes del cons tructivismo social, la experiencia fisica está parcialmente determinada

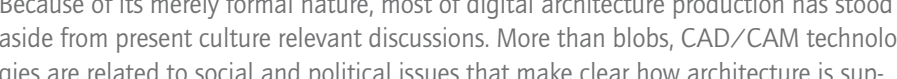 .}

por la cultura, particularmente por la cultura tecnológica: miramos lónica del momento, tanto literal como simbólicamente. Nuestras máquét nas y sus requerimientos específicos condicionan nuestros movimientos gestos cotidianos, complejizando la información que entrega la perce con. Desde esa perspectiva, el impacto del computador en la represetación debiera describirse no como un distanciamiento, sino más bien. siasmo depositado en el estado actual de la arquitectura digital, y la sime trica predisposición a su rechazo automático. Más que poner en discusión ation de agunos ejemplos referidos a esta arquitectura digital -de ahí más bien epistemon a réferencencias- me concentraré en asuntos de naturalezá su estado actual de desarrollo incipiente, nos sugiere respecto a las cambiantes categorías de la experiencia fisica? isi es que no está amenazada, cómo y porqué la definición de materialidad está evolucionando?

Cuando hablamos de producción arquitectónica, incluyendo el espectro

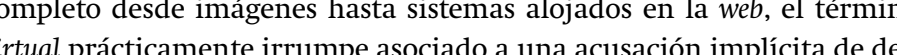
materialización, que opone declaradamente realidad virtual y realidad real. Sin entrar en el debate filosófico propio de estas ocasiones y las debiDolarización es extremadamente difícil de sostener en onservar que esta

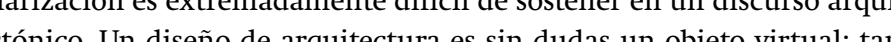
virtual que no solamente anticipa la construcción de un solo edificio, sino potencialmente la de una serie completa de variaciones y derivaciones. cón, que permite seguir diferentes vías para glegado a de indeterminausualmente uno de estos puntos terminales es el proyecto construide. zos por mejorar los protocolos y códigos del diseño de proyectos, de manera de controlar y anticiparse lo más posible a los

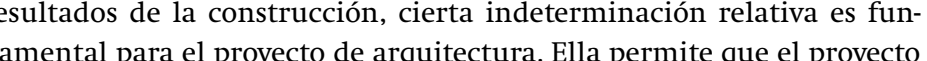
able, o más bien funcione como una matriz de posites que el proyecto lable, o más bien funcione como una matriz de posibles relatos, refer el proyecto no es más que un montón de copias de planos.

Volviendo a la pregunta por la materialidad, la situación se podría resumir así: mientras el proyecto se refiera a las realidades del entorno con Nuevamente. los dibujos y especificaciones evocan un Intro de nitentles -entendidos como un cierto ambiente o tono- más que una realidad material precisa y univoca, y reflejan por supuesto la ambigüedad de

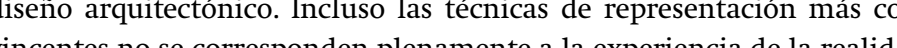

construida. Nunca vemos edificios en planta y elevación, ni menos en

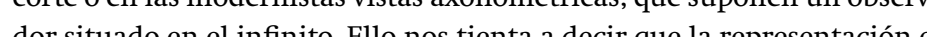
arquitectura, tal como en la cartografía, supone un observador situdo en un lugar imposible.

ta representación arquitectónica negocia entonces con tendencias opues tas: la búsqueda de la verosimilitud y el deseo de preservar cierto margen
de indeterminación. De hecho la necesidad de equilibrio entre estos dos ideales antagónicos da cuenta claramente de una paradoja inherente los dibujos arquitectónicos: mientras más especifico es el efecto fisico que se quiere lograr en el proyecto, más abstracta es su representación, como si esta tensión fundamental se tradujera en un equilibrio entre materia y abstraction. Desde es Ra este más elocuente que el juego de la luz en las distintas molduras y relieves de un edificio. Sin embargo, a menudo su representación era sorprendentemente distante del efecto que se quería lograr. Incluso en tratados canónicos, como los Cuatro libros de la arquitectura de Palladio, estas repre Considerando los antecedentes, ireals

las representaciones digitaetapa, la digitalización del diseño podría perfectamente parecer un mero avance de la técnica, un poder suplementario que se ha ofrecido al diseña digitales ban permitido a los arquitectos manipular for herramientas mente complejas y e imaginar con mayor libertad modificaciones durante la proyectación. Esta ampliación del vocabulario y la capacidad de inte racción en todas las etapas del diseño, ison realmente revolucionarias? ¿se trata más bien de un cambio cuantitativo o de un cambio cualitativo, dos con un set más grande y variado de lápices y escuadras?

isto por supuesto no es completamente cierto, desde que el computador terminó con la inmediatez del gesto manual en el proyecto. Entre a mano y la representación grafica se introdujo una capa de hardwarey

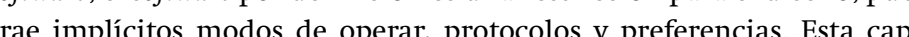
nueva equivale a la densa ausencia de herrramientas tradicionales en el estudio del arquitecto.

embargo, esta ausencia no es irremediable, gracias al desarrollo de cada vez más sofisticadas interfases que integran oficio y computador y dad. El Media Lab de MIT. ha invertido ãos de investigación en suathe digitales y pantallas táctiles, además de cámaras y sistemas de feedback controlados por láser que vinculan modelamiento digital y fisico. Sin

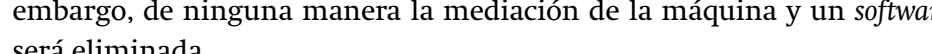

La diferencia entre el diseño producido a mano y el generado digitalzmiento en automóvil. La cuestión en ambos casos es la confrontací quina, donde ella no puede ser educida a un mero accesorio. Tanto el potencial del computador como espesor ro hacen distinto de otras herramientas tradicionales: su uso (a) posteriores a la II Guerra Mundial el automóvil ya había propiciado ese po de encuentros.

Otra posibilidad es considerar la asociación entre hombre y máquina yamo un nuevo ente compuesto, un hibrido mitad piel - mitad acero, que yer nonitor, mouse y teclado, podría interpretarse bajo esta luz de modo que arquitectura digital supondría la existencia de un autor cyborg. Esta roposición es sugerida por muchos autores contemporáneos dedicados estudio de las implicancias antropológicas de la tecnología computa (Picon, 1998)

lado estas consideraciones más amplias, la analogía con lautomóvil sigue siendo muy reveladora. La ya tradicional oposició itre la riqueza del caminar y la pobre experiencia del automovilista

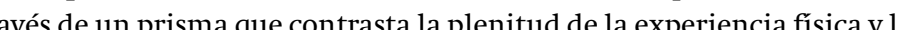
bstracción fomentada por un entorno tecnológicamente determinado. asi un siglo después de que el automóvil se transformara en el eje de cultura contemporanea, es un hecho que esta oposición no se aplica completamente a la experiencia del automovilista. Lejos de desmateria percepción de la materia: mi intención no es ahondar en una discusió detallada sobre estas transformaciones, sino más bien insistir en algunos puntos importantes.

Cuando conducimos no percibimos exactamente los mismos objetos que como lo vemos al caminar por la vereda A la velocidad del automón los objetos se reagrupan formando nuevas entidades perceptuales que podrían explicar en parte la escala y morfología del perfil urbano conmporáneo, como el paisaje que produce la rapida sucesio de carteles publicitarios a lo largo de una autopista.

nes corporales: aceleramuchas de estas sensaciones están inevitablemente golpaadas al uso de un motor; nos hemos acostumbrado tanto a la aceleración que tendemos a olvidar que su experimentación era prácticamente inalcanzable
para las anteriores sociedades no mecanizadas, donde los movimientos 
lentos y regulares eran la regla general. En un es casualidad que en lugares como el Media ambiente mecanizado, entre el entusiasmo Lab de M.I.T. prolifieren los intentos por rel por la velocitad y la perspectiva de un acci
dente se nos ofrecen simultineanente impresién des poder $y$ la de vulnerabililiad,
a famosa novela de James Graham Ballarar, La famosa novela de James Graham Ballard,
Crash, se concentra en este nuevo status ded cuerpo humano, o más bien en el hibridido
que resulta de la conjugacaín del cuerpo y su

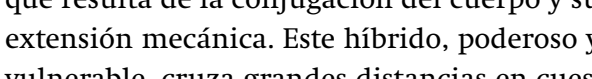
vulnerable, cruza grandes distancias en cuese
tion de minutos pero está constantemente. punto de estrellarse. Seguin Ballard, la mezcla de poder y vulnerabilitad tiene una fuerte
connotación sexuali i laccidente, el crassh fatal aue da titulo a la novela, se ha transformado
para sus personajes en una nueva forma, tec nológicamente mediada, del acto sexual
La redefinición perceptual de las enti.

La redeefinición perceptual de las entidades
con que nos relacionamos mientras nos desto plazamos en automóvil altera profundamerser nuestra noción del espacio, cambiando la condición existen naia d de nuestro cuerpo; quizís el
desplazamiento más significativo sea el sutil cambio que el uso del automóril produjo en
la experiencia cotidiana del espacio .1 automóvil ha a alterado, pero no disminuido, nueses
ra percepión fisica del mundo al modificar los limites y la sustancia de lo material.

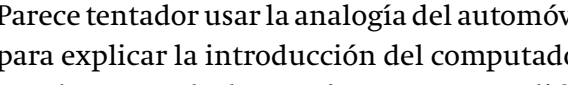

en el campo de la arquitectura, entendién dolo como otro vehículo que induce un nuevv
desplazamiento en la experiencia fisica y desplazamiento en la experiencia fisica y
materia. Indiriectamente, el arquitecto ass tido por computadador recuerda a un conductor
pasajero, embarcando en un vivije que le per-

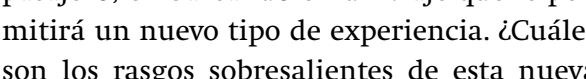
son los rasgos sobresalientes de esta
experiencia en el mundo material?

El computador nos presenta nuevas entide. des perceptuales y objetos. Si el arquitecto antes manipulaba formas estaticas, aho
puede trabajar con fluidos geomentricos. Lh deformaciones de volúmenes o de super
cies pueden registrarse de manera muy pret des pueden registrarase de manerar myy pifeos,
cisa, cosa imposible para los medios graficos de representación usados anteriormente; de
hechoo estas variacioiones pueden ser generadas $y$ controladas en el monitor en tiempo real
Curiosamente, el empleo de medios digitales en la proyectación es muy cercano a prácticas como el modelado en arcilla o phsticines $\begin{array}{ll}\text { que en lugares como el Media } & \text { retorcida que parece un pedazo de papel o de } \\ \text { roliferen los intentos por rela- } & \text { tejido arrugado. Las imágenes siguientes reve- }\end{array}$ lan que se trata de un proyecto enorme, cas
una megaestructuras. El imaginario comp tacional está en profunda concordancia co un mundo organizado a partir de fractales bs este ambiente infometria tradicionalen este ambiente información y complejidad 政 la posibilidad de nuevos registros de fll los geométricos. El proyecto de Reiser
Umemmoto ${ }^{3}$, West Side Convergence (Nueva geometrías que, congelado, origina una
cifrar ) el objeto de proyecto.

$\begin{array}{ll}\text { forma arquitectónicá. } & \text { Además de los desafios planteados para la idea } \\ \text { Además de las deformaciones y movimien- } & \text { de escala, las tennologias digitales han dismi- }\end{array}$ of el computador permite la manipulación nuido certa claridad alcanzada en el pasado conjugados con cualidades como la textura tónica y su materialización. Esta disociación adquieren la categoría de cuasi-objetos para el es evidente en las complejas superficies dise arquitecto. En estas simulaciones, los pará- ñadas por Frank Gehry con el software Catia e puede intensificar, atenuar, difuminar, raciones estructuraless. A pesar de las dife controlarse según amplios patrones de com- existe entre la diáfana y luminosa maqueta binación, mezclando grados de rugosidad, presentada por Toyo Ito para el concurso de
pulido, capacidad reflectante y transparenin al punto de hacerlas prácticamente tác- ral de las pesadas placas de acero sestructutia al punto de hacerlas prácticamente tác-
tiles. Estas de las pesadas placas de acero usadas para $\begin{array}{ll}\text { múltiples manifestaciones, desde efectos } & \text { de Yokohama de Foreign Office revela una } \\ \text { turerficiales como el mapeo y la proyección } & \text { tensión parecida entre la fluidez del diseño }\end{array}$ $\begin{array}{ll}\text { superficiales como el mapeo y la proyección } & \text { tensión parecida entre la fluidez del diseño } \\ \text { de hipersuperficies -posibilitadas por la capa- } & \text { original y las técnicas movilizadas para con }\end{array}$ idad del entorno digital para una imagen en la textura de cualquier objeto través de un computador no sólo es complejo mente comprobadas de Bernard Cache y su está lleno de sorpresas por el salto que fre estudio Objectile en París.

tura digital (como el control de superficies) aportes esenciales para la disciplina, La oposición de críticos como Frampton está otras aparecen más problemáticas y difu- directamente relacionada al reconocimiento
sas. En el caso del automóvil, la aparición
de esta distancia entre la representación por $\begin{array}{lll}\text { de nuevas entidades relacionadas a él fue } & \text { medios digitales y la materialización del pro- } \\ \text { acompañada por la pérdida del sentido de la } & \text { yecto. No obstante y pese a lo pettubs }\end{array}$ certurbad dad. En el caso de los medios digitales y la riamente sinónimo de una desmoterializecsionarquitectura, esa pérdida principal es la escala, de la arquitectura; el computador redefine
a dejó de ser evidente. icuál es la verda- la materialidad, más que abandonarla por que dejó de ser evidente. ¿Cuál es la verda- la materialidad, más que abandonarla por

monitor de un computador? A pesar de la Estos desplazamientos demandan una rede
hoclusión de figuras a escala en fotomontajes, finición de los procedimientos y objetivos amenudo resulta difícil contestar esta pre- del diseño de proyectos. La realidad digita unta. La presentación tipo de un proyecto necesita de una práctica visual renovada, que como Beachness (Amsterdam, 1997) de Nox es pueda moverse con soltura en el complejo principio, sólo es legible un complejo labe- y lo local, entre la definición general del pro- cambios formales producidos por variacio- lelismo de diferentes escenarios; al mismo las piedras, cuchillos y hachas que ampliaron cambio más pequeño puede afectar el total al diseñador en la toma de decisiones resteoría del caos proclama que el aleteo de que los medios digitales posibilitan. Por su una mariposa puede desatar una tormenta a cercanía al territorio conceptual, y por invovarios kilómetros de distancia (Gleick, 1987) ta sensibilidad generada por la dependencia mente encuentra analogías en la sensación de un conductor que maneja a toda velocidad sobre una superficie irregular, donde e obstáculo màs insignificicante puede causar consecuencias catastró́icas. Marcos Noval digital: "las operaciciones asociadas a la idea de un
ingudo sugieren que la parametrización conduce líquido sugieren que la parametrización conduce a
una variabilidad radical que se produce dentro de na continuidad determinada por una cosa y su han adentrado en un mundo eminentemente dinámico y fluido, que da especial intensidad $a$ algunas de las sensaciones que experimen-
tamos $y$ a las decisiones que ellas informan. El automóvil es una metáfora, y como tal no su trayectoria lineal, el mundo digital que se
despliega ante los ojos del diseñador es multidimensional: fluye teóricamente en todas direcciones $\mathrm{y}$ es teóricamente reversible. necesaria secuencia del proceso de diseño, que parte con los esquemas preeliminares $y$ que termina con la entrega de las especifica-
cones técnicas definitivas, involucrando ciones técnicas definitivas, involucrando a
numerosos agentes (desde los colaboradores del arquitecto hasta los especialistas). En otras palabras, el diseño asistido por computador
no puede ser un laberinto de exploraciones no puede ser un laberinto de exploraciones frece la dupla software-hardware. Mientras ciones sin fin, hay un punto en que debe producirse un corte para optar por una de ellas esa toma de decisión tiene que orientarse a romper
la teóricamente reversible naturaleza de la mani-

la teóricamente reversible naturaleza de la mani-
pulación digital. nueva actitud del proyectista, basada en eva luaciones estratégicas del potencial evolutivo del proyecto en ciertas etapas críticas de su uso del computador supone cierto estado de humano ha estado determinado por una
reffexión, basado en la comparación y para-
gradual externalización de funciones, desde crar la supresión de detalles innecesarios, esquemas puramente mentales; esta aproxinación es completamente inconsistente con a verdadera naturaleza del diagrama, consderando el hecho que son inseparables de los especie de condición fisica propia, similar a la entemente abstracta usada por (1) presionante paralelo entre los diagramas rquitectónicos contemporáneos -a menudo gunos diagramas geopoliticos producidos a principios del s. XX (Raffestin, 1995). Ambos e basan en una descripción esquemátic del mundo, que tiende a obviar las comple hablar de la especificidad historicica). La geopoen el mismo sentido, están basados en agrupaciones masivas $y$ datos globales?. En ambos
casos el mundo es visto como un campo de anifestación de fuerzas más que como una geo grafí estática: tal como en la geopolíitica, los diagramas arquitectónicos contemporáneos usan abundantes flechas y gráícos tratando
de hacer visibles estas fuerzas, que convergen de hacer visibles estas fuerzas, que convergen
en nudos que pueden ser vistos como objetivos o blancos. En ambos casos, lo que parece estar en juego es la aprehensión por la acción
cntinua que demandaría un entorno que hye y se mueve constantemente.

( úper-memoria o una avanzada herramienta para la exploración lógica. El antropólogo francés Leroi-Gourhan, por ejemplo, rastrea humano a través del uso de herramientas teca gicas en Le Geste et la parole, un libro que desde las primeras piedras cortadas y pulidas hasta los primeros computadores (Leroiel computador. computador puede indudablemente ser
sto como una extensión de la mente, pero ambién altera nuestra percepción de los aciones. Nuevas interfases actualmente desarrollo afectarán nuestras habilidades motoras, aunque ya el mouse produjo la aparición de nuevos gestos. Entre los adolescentes enso extendido de videojuegos ha producido el desarrollo de un gran nuin
cada vez más específicos. ta propia percepción del espacio se verá a
vez afectada por estos cambios fisicos. Peliculas como Johnny Mnemonic, The matrix o Whority report han imaginado cambios en la esarrollo de sofisticadas espacio fisico y digital. Aunque esta hibrida coón no está completamente desarrollada gespectos de este cambio de la noción materia ya son evidentes.

ma, los códigos visuales están cambiando a gran velocidad. Ya no nos sor-
prende la posibilidad que entregan los medios digitales con efectos como el zoom in y zoom
unt. Más bien tendemos a probiri el . inos, como si la realidad fuera resultas té de un compromiso temporal o de un enfoque de mediano alcance entre un lente muy grande uno muy pequeño. Los objetos y formas ces a medio camino entre las superficies $y$ exturas que (como vistas desde muy cerca) evocan alguna clase de arte abstracto, y otras vistas menos abstractas, similares a las imáge uperficies y texturas. En ambos casos la perde estas dos clases de pieles o superficies.

estado actual de la forma y el objeto puede que generó la la globalización. La ga globalización puede entenderse como un particular circuito cerrado que conecta directamente elementos locales con otros generales, prescindiendo de

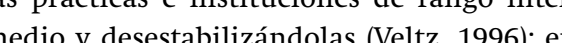
nuestro mundo global vemos las cosas desde 
$\begin{array}{llll}\text { muy cerca o desde muy lejos, y no es acciden- } & \text { Jesse Reiser y otros? Su arquitectura de blobs } \quad \text { La segunda razón para confiar en la nueva }\end{array}$

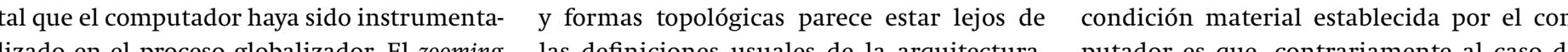
lizado en el proceso globalizador. El zooming las definiciones usuales de la arquitectura, putador es que, contrariamente al caso del de la noción tradicional de escala, implicita - separa el mundo de las simulaciones compu- aislada, como el individuo tecnolósico señalado en las prácticas digitales y en la globalización, tacionales de la percepción cotidiana de los por el filósofo francés George Simondon, una

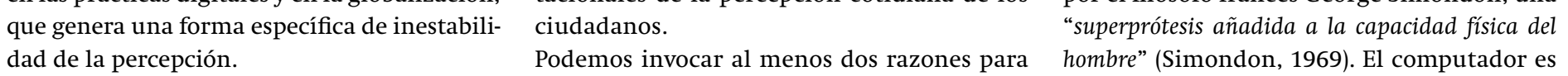
$\begin{array}{llll}\text { dad de la percepción. } & \text { Podemos invocar al menos dos razones para } & \text { hombre" (Simondon, 1969). El computador es } \\ \text { Tal inestabilidad borra la distinción entre abs- } & \text { esperar una respuesta optimista al respecto. } & \text { sólo una parte del universo digital slobal eve }\end{array}$ que generan lecturas a partir de las catego- el computador sigue permeando la vida coti- diales y también millones de unidades perrías usuales de forma y objeto. En la era del diana, por lo que estos cambios en la noción sonales. Se podría contraargumentar que el computador, la fisica de los sólidos y la mani- de lo material podrian entenderse como un auto es a su vez inseparable de un universo de
pulación de ADN, la materialidad progresiva- fenóner $\begin{array}{llll}\text { pulación de ADN, la materialidad progresiva- } & \text { fenómeno general y transversal. Supuesta- } & \text { semáforos, autopistas, estaciones de servicio } \\ \text { mente se ha ido definiendo en la intersección } & \text { mente, todos estaríamos llamados a habitar } & \text { yedificios de estacionamientos; sin embargo, }\end{array}$ de dos categorías aparentemente opuestas: tanto el mundo concreto y ordinario como el este es más bien un sistema de partes contauna totalmente abstracta, basada en signos mundo virtual; de ahí la famosa declaración bles y conectadas antes que un tejido con$\begin{array}{llll}\text { o señales, y otra ultra-concreta que involucra } & \text { de Toyo Ito respecto a que los arquitectos } & \text { tinuo y sin costuras. La densidad y los altos } \\ \text { una presisa y asi patológica descripción del } & \text { debieran proyectar para habitantes dotados } & \text { niveles de interconectividad }\end{array}$ una precisa y casi patológica descripcionn del debieran proyectar para habitantes dotados niveles de interconectividad y redundancia yla textura, reveladas por las posibilidades del la era moderna tenemos una doble corporeidad. El difícil describirlo en términos de un sistema, lo ultramaterial representa el nuevo mundo una serie de fluidos que recorren su interior, yel rio o paisaje parezcan más apropiadas para su de sensaciones y movimientos en el que hoy En el campo disciplinar

Ley ya es común la coexistencia arquitectura de naturaleza diagramática con un renovado interés en algunos de los aspectos materiales mas concretos. Este fenomeno se da tambieit que representa la cercanía entre lo abstracto y lo concreto. Usando un dispositivo GPS nos conectamos tanto a una grilla geodésica
global y abstracta como a nuestro alrededor inmediatos. Mientras el computador ha ambiente digital eventualmente modificará el herramientas como el GPS pueden ayudar redefinir los proble
secuencias urbanas.

Pero aún queda pendiente una pregunta ¿Cómo las intuiciones del arquitecto o de urbanista pueden traspasarse al públice
que habita sus proyectos? En otras palabras, que habita sus proyectos? En otras palabras,
ccómo los asuntos de la nueva materialidad anhelada por los arquitectos que experimen-
tan con medios digitales, pueden interesar un público más amplio que la mayor parte
del tiempo no tiene ninguna referencia

de las variadas (y también contradictorias) cuerpo virtual, relacionado al mundo a thor, y fujo de electrones" "Ito, 1997). En realidad, estos te de lo que constituye, hoy por hoy, la lidad, este territorio digital enter as placas de acero que recuerdan enormes pasiva. Tal como han señalado otos manera navales, y al mismo tiempo la revolución digital es -ene una componente fluida y una lumino- una revolución del material, en cuanto a los $\begin{array}{ll}\text { cidad que la hacen ver como una piedra pre- cambios en la manera en que los producimos } \\ \text { ciosa electrónica. En este caso y lejos de ser } & \text { y usamos. En Harvard Graduate School of }\end{array}$ accidental, el salto entre materia y represen- Design, un grupo de profesores y estudianMencioné la intluencia de los videojuegos $\quad$ expresión arquitectónica (Mori, 2002), asunto en las nuevas generaciones, cuyo comporta- que ya había sido investigado por diseñadofiguras de enanos, princesas y do figuras de enanos, princesas y ogros que La producción de materiales con ayuda de
corren y saltan en la pantalla de un Game- computadores pareciera anular la distancia boy. Esta generación ha desarrollado actitu- entre representación y materia, dado que la des mentales y fisicas que demandarán un definición de materialidad se hace en térmisevo tipo de espacio. ne espacio que puede nos diferentes a la tectonica tradicional. Pero sistemas de pistas y una serie de escenarios prodactucida por la eliminación de la compleja que se despliegan sucesivamente, en vez del serie de interfases necesarias para salvar la tradicional sistema de mapas holísticos. Las distancia entre representación arquitectó$\begin{array}{lll}\text { expectativas espaciales de tal generación } & \text { nica y diseño de materialidad; el compu- } \\ \text { podrían perfectamente ser satisfechas sólo } & \text { tador está lejos de eliminar esa distoris }\end{array}$ por una arquitectura comprometida con el $\begin{array}{lll}\text { por una arquitectura comprometida con digital. } & \text { tro continuo y documentado del proceso }\end{array}$ que va desde la representación pura hasta
las especificaciones técnicas. En su revolulas especificaciones té́cnicas. En su revolu-
cionario curso de Geometría descriptiva,
Gaspard Monge comenzaba distinguiendo dos grandes grupos de objetos, dependiendo
si eran susceptibles o no de una definición si eran susceptibles o no de una definición
rigurosa?. En la era digital, sin embargo, es rigurosa'. En la era digital, sin embargo, es
posible definir con rigurosidad cualquier posible definir con rigurosidad cualquier
objeto o material, en cada uno de sus estados posibles: la verdad la generalización de la proyectación como una práctica que ya no es aplicable sólo a
edificios y sus múltiples sistemas técnicos, edificios y sus múltiples sistemas técnicos. sino también a materiales e incluso a la
naturaleza como una realidad manipulada. Muchas de las propuestas contemporáneas del paisajismo, como los proyectos presenta-
dos al concurso Fresh Kills landfillio, no consi-
deran más a la naturaleza como un recurso deran más a la naturaleza como un recurso cada vez producto susceptible de controlar a través de un diseño apropiado. La extension del uso del término lanascape urbanism respecto a situaciones de proyecto como Downsview
o Fresh Kills aparece como consecuencia de esta tendencia (Waldheim, 2002). En una naturaleza tecnologizada, el diseño permea completamente la materialidad. A pesar de tectónica y los ele la representación arquiverdadera novedad no es el aumento de la distancia entre el proyecto y la materia; más
bien es la existencia de una profunda inte bien es la existencia de una profunda interacción, que eventualmente podría poner en
jaque la identidad profesional tradicional de jaque la identad profesional tradicional de
arquitectos $\mathrm{e}$ ingenieros. Es más, estos roles fueron construidos sobre el supuesto de una
distancia entre el mundo físico y el intelectual, que el diseño estaba destinado a salvar, minación entre lo abstracto y lo concreto. estas identidades no pueden permanecer inmutables. Las declaraciones del ingeniero Cecil Balmond respecto a su compromiso y participación total en los procesos de diseño, alejadas del usual confinamiento de su
especialidad al ámbito del cálculo estructural, representan nuevas perspectivas que se abren en un mundo que diluye la distinción
entre abstracción matemática y espacialidad

La potencial aplicación generalizada de los
procesos propios del diseño nos hace esponsables que nunca por sus consecuenás como una minco sera visto cada vez naturaleza, materiales y edificios. Por lo to, estamos ante la cuestión de una nueva ara los arqui(a) sus realizaciones: para ins que acarrean y culturales ya no es sufientes economicas anford Kwinter una vez, considerar que el "las fluctuaciones de las condiciones histomar "Is fluctuaciones de las condiciones históricas Como hemos visto, hoy materialización signica mucho más que el mero entendimiento de las fuerzas que determinan el mercado Jeb resto de los ciudadanos deben tom tor reciticectos

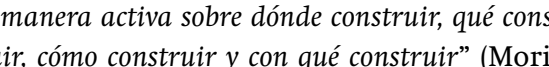
2002). Debería agregarse a la lista "y cuándo lo o sustentable y el medio ambiente se han
convertido en asuntos cruciales. Abstenerse e iniciar una construcción es cada vez más menudo una solución mejor que comprometerse con desarrollos que podrían dañar

apinión, el real problema de la arquidesmaterialización, sino más bien su falencia especto a una agenda política y social clara que nunca. El éxito creciente de arquitectos (a) ajo en el desarrollo de estructuras sustentauna pia explicarse muy bien en función innovación teconológica articyaterialidad y discurso político y social claro y consistente. da del proyecto arquitectónico, la materialiominante para la disciplina preocupación dominante para la disciplina, pero ahora
además se ha convertido en sinónimo de
una nueva responsabilidad. Es un frente en plena evolución, cuyas implicancias y signi-
ficados aún no están claros; en este sentido, vez una de las tareas urgentes sería ilumof

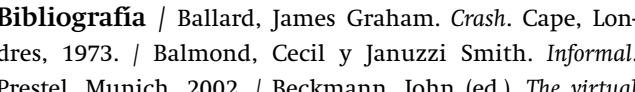

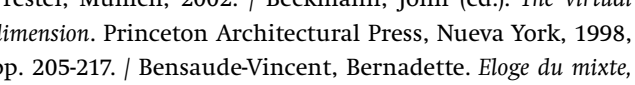

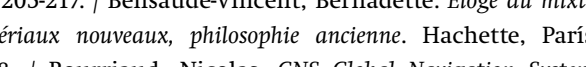

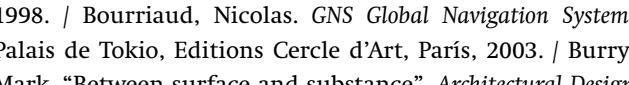

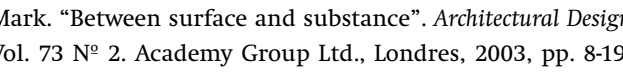
Cache, Bernard. Earth moves: The furtisting of territories

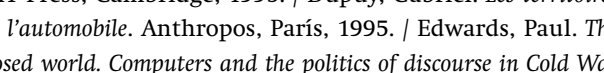

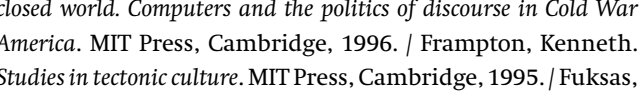

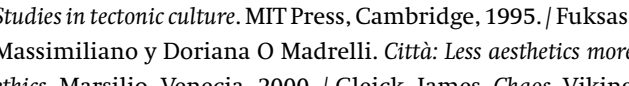

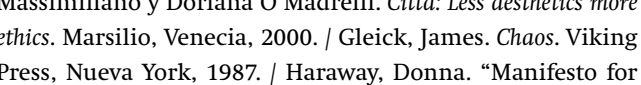

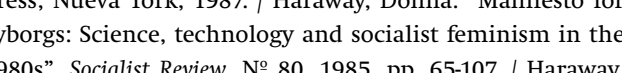
Donna. Simians, $y$ borgs and women. The reinvention of nature Moutledge, Nueva York, 1991. I Ito, Toyo. "Tarzans in the
media forest". $2 G$ Vol. II. Editorial Gustavo Gili, Barcelona. 1997, pp. 121-144./ Koolhaas, Rem; Boeri, Steffano; Kwinter,

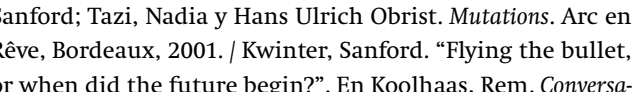
wns with students. Princeton Architectural Press, Nueva Yorl, 1996. / Langins, Janis. La République evvait besoin de Savant
les debuts del

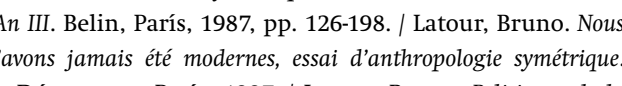

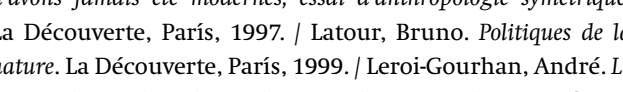

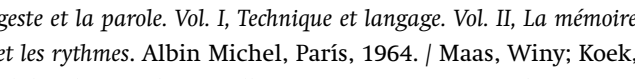
chardy J Jacob van Rijs. Farmax. Exarsisins on density. 1010

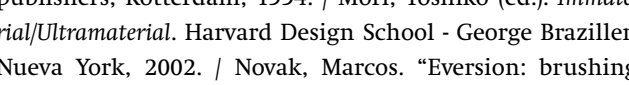

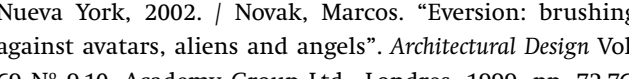
6 № 9.10. Academy Group Itd., Londres, 1999, pp.7 $72-76$
Perrella, Stephen. "Electronic baroque, Hypersurface

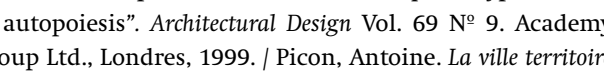

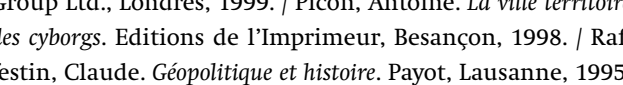
Simondon, George. Du mode dexistence des bjoftst techniqueses

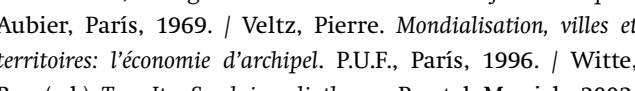
Waldheim, Charles. "Landscape urbanissm: a genealogy"

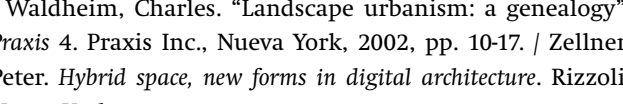
Peter. Hybrid space, new forms in
Nueva York, 1999, pp. 114-117. 